\section{Schwellenwert der ROC}

R.-D. Hilgers ${ }^{1}$, N. Heussen ${ }^{1}$ und S. Stanzel ${ }^{2}$

${ }^{1}$ Institut für Medizinische Statistik, Universitätsklinikum der RWTH Aachen, Aachen, Deutschland

${ }^{2}$ DKFZ Heidelberg, Heidelberg, Deutschland

Synonym(e) Cut-off-Wert der ROC

Englischer Begriff cut-off value; cut-off

Definition Der Schwellenwert der ROC ist ein Wert zur Klassifizierung von quantitativen Messergebnissen einer - Stichprobe in diagnostisch positive bzw. negative Fälle.

Beschreibung Um zu einer Klassifizierung der Messergebnisse eines diagnostischen Testverfahrens $(\triangleright$ Test, diagnostischer) in positiv, für nicht normale Werte, und negativ, für normale Werte, zu gelangen, wird der Wertebereich dichotomisiert. Dazu wird ein Schwellenwert gewählt, anhand dessen der Messbereich in 2 nicht überlappende Bereiche (positives/ negatives Testresultat) geteilt wird.

Im Rahmen diagnostischer Testverfahren besteht eines der Ziele der ROC-Analyse darin, einen geeigneten Schwellen- wert zu ermitteln. Aufgrund der Eigenschaften der $>$ ROCKurve empfiehlt sich vor allem ein Wert nahe der linken oberen Ecke der grafischen Darstellung. Sollten nicht klinische oder praktische Gründe für einen bestimmten Schwellenwert sprechen, etwa ein Schwellenwert mit einer geringen falsch-positiv oder falsch-negativ Rate, so könnte man auch alternativ den Ausdruck [Sensitivität $-\mathrm{m} \times(1-$ Spezifität $)$ ] maximieren. Ist $\mathrm{m}=1$, so maximiert man lediglich die Summe aus Sensitivität ( $\triangleright$ Sensitivität, diagnostische) und Spezifität ( $\triangleright$ Spezifität, diagnostische). Dies entspricht der Minimierung der Wahrscheinlichkeiten möglicher Fehlentscheidungen, was in der Praxis nicht notwendigerweise die einzig zu bevorzugende Strategie darstellt. Bei der Wahl von $m$ können daher auch Kostenargumente für Falschentscheidungen und A-priori-Wahrscheinlichkeiten (s. D A priori odds) Berücksichtigung finden.

\section{Literatur}

Hilgers R-D, Bauer P, Scheiber V (2002) Einführung in die Medizinische Statistik. Springer, Berlin/Heidelberg/New York

Zweig MH, Campbell G (1993) Receiver-operating characteristic (ROC) plots: a fundamental evaluation tool in clinical medicine. Clin Chem 39:561-577 